\title{
BRONCHIAL ASPIRATES GLUCOSE LEVEL AS INDICATOR FOR METHICILLIN-RESISTANT STAPHYLOCOCCUS AUREUS (MRSA) IN INTUBATED MECHANICALLY VENTILATED PATIENTS
} By

\author{
SHERIF ALSAYED ${ }^{1}$, SAMAR MARZOUK ${ }^{2}$, ESSAM MOUSA ${ }^{3}$ AND ASHRAF \\ RAGAB $^{4}$
}

Department of Chest, Faculty of Medicine, El Fayoum University ${ }^{1}$, Department of Biochemistry, Faculty of Medicine, Cairo University ${ }^{2}$, Department of Internal Medicine, Faculty of Medicine, Al Azhar University ${ }^{3}$ and Department of Anesthesia, Faculty of Medicine, Cairo University ${ }^{4}$, Egypt

\begin{abstract}
This study evaluated if the level of glucose in bronchial aspirate serves as indicator for the risk of MRSA infection in intubated mechanically ventilated ICU patients.

A total of 50 critically ill patients was enrolled and were under tight glycemic control to abolish the effect of hyperglycemia on bronchial secretion, if they were expected to require mechanical ventilation for more than 48 hours. Bronchial aspirates were detected for glucose and sent twice weekly for microbiological analysis and whenever an MRSA was expected.

The results showed that all the patients had glucose tested in bronchial aspirates. Glucose was detected in bronchial aspirates of 28 of the 50 patients. Glucose in bronchial aspirates in these patients ranged between $(2.9-5.1 \mathrm{mmol} / 1)$. MRSA was detected in 22 patients where 28 were MRSA free of the MRSA patients 19 had positive glucose where glucose was positive in 28 patients of them 19 (86.4\%)where MRSA positive to 9 with no MRSA (32.1\%).The risk of having MRSA present markedly increased significantly in the presence of glucose: ( $\mathrm{p}$ value .001)

Key words: Glucose level, Indicator, Methicillin-resistant Staphylococcus aureus, Ventilated patients.
\end{abstract}

\section{Introduction}

Although $S$. aureus has been causing infections (staph infections) probably as long as the human race has existed, MRSA has a relatively short history, was first noted in 1961, about two years after the antibiotic methicillin was initially used to treat $S t$. aureus and other infectious bacteria (Jones, 2010). The resistance to methicillin was due to a penicillin-binding protein coded for by a mobile genetic element termed the methicillin-resistant gene (mecA). Nowadays, the gene has continued to evolve so that many MRSA strains are currently resistant to several different antibiotics and is sometimes termed a superbug because of its ability to be resistant to several antibiotics (Kock et $a l, 2010)$. This organism is known for causing skin infections in addition to many other types of infections. There are other designations in the scientific literature for these bacteria according to where the bacteria are ac- quired by patients, such as communityacquired MRSA (also termed CA-MRSA or CMRSA), hospital-acquired or health-careacquired MRSA (also termed HA-MRSA or HMRSA), or epidemic MRSA (EMRSA). Data supplied by the CDC in 2011 suggest this number has declined by about $54 \%$ from 2005 to 2011, in part, because of prevention practices at hospitals and home care. In addition, hospital deaths from MRSA infection have declined by about 9,000 per year from 2005-2011. However, the CDC recently estimated about 80,000 infections with 11,000 deaths occurred in 2011, but a high rate of nasal carriage and multidrug-resistant St. aureus was found, indicating the need for standard infection control to prevent transmission in our health care setting (Shibabaw et al, 2014).

More than $70 \%$ of St. aureus isolated in ICUs is MRSA Many patients not infected on admission may actually become colo- 
nized with MRSA while in the ICU (Hill et $a l, 2013)$. Analysis of a large surgical ICU cohort showed that $8 \%$ of patients were colonized with MRSA at the time of admission, and that these subjects can serve as a reservoir for the spread of this pathogen. Beyond the hospital, traditional nosocomial strains of MRSA are increasingly implicated as the cause of "healthcare-associated" infections (Robotham et al, 2011).

The bacterial epidemiology of VAP depends on a panel of factors including mechanical ventilation duration, length of hospital and ICU stays, previous exposure to antimicrobials, local epidemiology and potential epidemic phenomenon in a given ICU. Rapid changes in the oropharyngeal flora of intubated patients (even in the absence of antibiotic exposure) represent a key determinant (Hyllienmark et al, 2012) as micro aspirations of pharyngeal secretions constitute the leading physiopathological mechanism of VAP, early-onset VAP; within the first 4 days of mechanical ventilation (Jones, 2010). However, MDR pathogens may be isolated in early-onset VAP when risk factors exist prior to ICU admission, and even when such risk factors are lacking [notably for Pseudomanas aeruginosa and methicillin-resistant [notably for Pseudomonas aeruginosa and methicillin-resistant St. aureus (Tacconelli and De Angelis, 2009) enlightening the limits of this classification in the current context of bacterial resistance.

Philips et al. (2003) reported that glucose was detected in bronchial aspirate of critically ill patients with stress hyperglycemia when the blood glucose concentrations had increased to $(>6.7-9.7 \mathrm{mmol} / \mathrm{l})$. The increased glucose concentrations in the airways can increases the risk of chest infections, either by providing added nutrition for bacteria or by interfering with normal immune functions (Philips et al, 2005).

Van den Berghe et al. (2001) found that on cardiothoracic intensive care unit (ICU), mortality was decreased by control of blood glucose to normal limits $(4.4-6.1 \mathrm{mmol} / \mathrm{l})$ also). This was principally through a reduction in episodes of sepsis, but no mechanisms for this effect were suggested. The normal airways secretions contain less than $0.5 \mathrm{mmol} / 1$ glucose; lower limit of standard methods of detection (Wood et al, 2004).

The aim of this study was to detect the relation between the presences of glucose in bronchial secretion of intubated mechanically ventilated patients and MRSA infection

\section{Subjects, Materials and Methods}

Fifty critically ill patients requiring intubation and ventilation were chosen from the New and Old Jeddah Clinic Hospitals. With age ranging between 30 and 80 years old) Patients were included if they were expected to require intubation for more than 48 hours. Patients were excluded if they had had a previous ICU admission during this hospital admission in the period March 2013 to March 2014. Nineteen patients were admitted for respiratory reasons, five for cardiology reasons, and five for gastroenterology reasons, one for hematological problems, and 15 for neurological reasons, five for infectious reasons, and four for general medicine problems. Severity of illness was measured using the sequential organ failure assessment (SOFA) system. Outcome was assessed at discharge from the ICU and after 6 months

All patients were subjected to the following: 1- Detailed history and thorough medical examination, 2- Chest $\mathrm{x}$-ray, CBC, Blood glucose and were under tight glycemic control aiming at blood glucose level between 90-144 mg/dl (Van den Berghe et al, 2001), 3- Bronchial aspirate analysis were collected by a bronchial aspirate with fiber optic bronchoscopy using a large channel bronchoscopy (Olympus BF 1T-20D; Olympus, New Hyde Park, NK, USA. The fiberoptic bronchoscopy (FOB) was inserted through the endotracheal tube via a steri connector (Carden Swivel Connector, Bivona, Griffith Microscience Inc, IN, USA and glucose analyzed and sent twice weekly for microbiological analysis and whenever an infection 
was suspected. Glucose was measured simultaneously in arterial blood using a near patient glucose analyzer (vitros 250, Johnson and Johnson co). Bronchial aspirates were analyzed for glucose at the bedside using a glucose oxidase stick, followed by more precise glucose analysis of bronchial aspirate filtrate and bronchial aspirates; Glucose appeared in bronchial aspirates as blood glucose levels exceeded 6.7-9.7 mmol/1 (Murray and Washington, 1975).

Microbiological methods: Samples for microbiological evaluation were collected in sterile receptacles. Sample quality was evaluated (Murray and Washington, 1975) and samples were immediately seeded on reaching the laboratory. $S$. aureus colony counts of $>103 \mathrm{cfu} / \mathrm{mL}$ were considered to be significant. Quantitative culture was performed on arrival of the samples at the laboratory. $S$. aureus at $>105 \mathrm{cfu} / \mathrm{mL}$ was considered significant (Somerville et al, 2002).

Statistical analysis: Data were described in terms of mean \pm standard deviation $( \pm \mathrm{SD})$, or frequencies (number of cases) and percentages when appropriate. Comparison of numerical variables between the study groups was done using Student $t$ test for independent samples in comparing normally distributed data and Mann Whitney $U$ test for independent samples when data not normally distributed. For comparing categorical data, Chi square $\left(\chi^{2}\right)$ test was performed. Exact test was used instead when the ex- pected frequency is less than 5. $p$ values less than 0.05 was considered statistically significant. All statistical calculations were done using computer program SPSS (Statistical Package for the Social Science; SPSS Inc., Chicago, IL, USA) version 15 for Microsoft Windows

\section{Results}

Of the 50 patients, 32 were men and 18 women. All 50 patients had glucose tested in bronchial aspirates. Glucose was detected in bronchial aspirates of 28 of the 50 patients. Glucose in bronchial aspirates in these patients ranged between $(2.9-5.1 \mathrm{mmol} / \mathrm{l})$. MRSA was detected in 22 patients where 28 were MRSA free of the MRSA patients 19 had positive glucose where glucose was positive in 28 patients of them 19 (86.4\%) where MRSA positive to 9 with no MRSA (32.1\%).The risk of having MRSA present was markedly increased significantly in the presence of glucose: ( $p$ value 0.001). There were no significant correlation between incidence of MRSA and length of stay in ICU ( $p$ value 0.015 ), age of the patient ( $p$ value 0.969 ), diabetes mellitus ( $p$ value 0.254 ), chest infiltrates ( $\mathrm{p}$ value 0.302 ), or leucocytes' count ( $\mathrm{p}$ value 0.080 ). There was no correlation between age of the patient and blood glucose level in bronchi ( $p$ value $0.740)$. Details were given in tables $(1 \& 2)$ and figures $(1,2 \& 3)$.

Table 1: Demographic, clinical and lab parameters between the study groups

\begin{tabular}{lccc}
\hline \multicolumn{1}{c}{ Item } & $\begin{array}{c}\text { MRSA } \\
(\mathrm{n}=22)\end{array}$ & $\begin{array}{c}\text { No MRSA } \\
(\mathrm{n}=28)\end{array}$ & P value \\
\hline Age (mean \pm SD, years) & $57.0 \pm 16.3$ & $58.9 \pm 9.4$ & 0.969 \\
DM [count $(\%)$ ] & $16(72.7 \%)$ & $16(57.1 \%)$ & 0.254 \\
TLC (mean \pm SD, x 1000/cmm) & $11.3 \pm 5.3$ & $16.3 \pm 9.8$ & 0.080 \\
Glucose in bronchial lavage [count $(\%)]$ & $19(86.4 \%)$ & $9(32.1 \%)$ & $<0.001$ \\
CXR infiltration [count $(\%)]$ & $6(27.3 \%)$ & $4(14.3 \%)$ & 0.302 \\
ICU stay (mean \pm SD, days) & $20.7 \pm 18.2$ & $9.04 \pm 7.3$ & 0.015 \\
\hline
\end{tabular}

Table 2: Comparison of age in years between cases with and without glucose in brochial lavage (n-22)

\begin{tabular}{|c|c|c|c|}
\hline Item & With glucose in brochial lavage & without glucose in brochial lavage & $\mathrm{P}$ value \\
\hline Age $(\mathrm{M}+\mathrm{S})$ & $57.5 \pm 13.4$ & $58 \pm 12.2$ & 0.740 \\
\hline
\end{tabular}




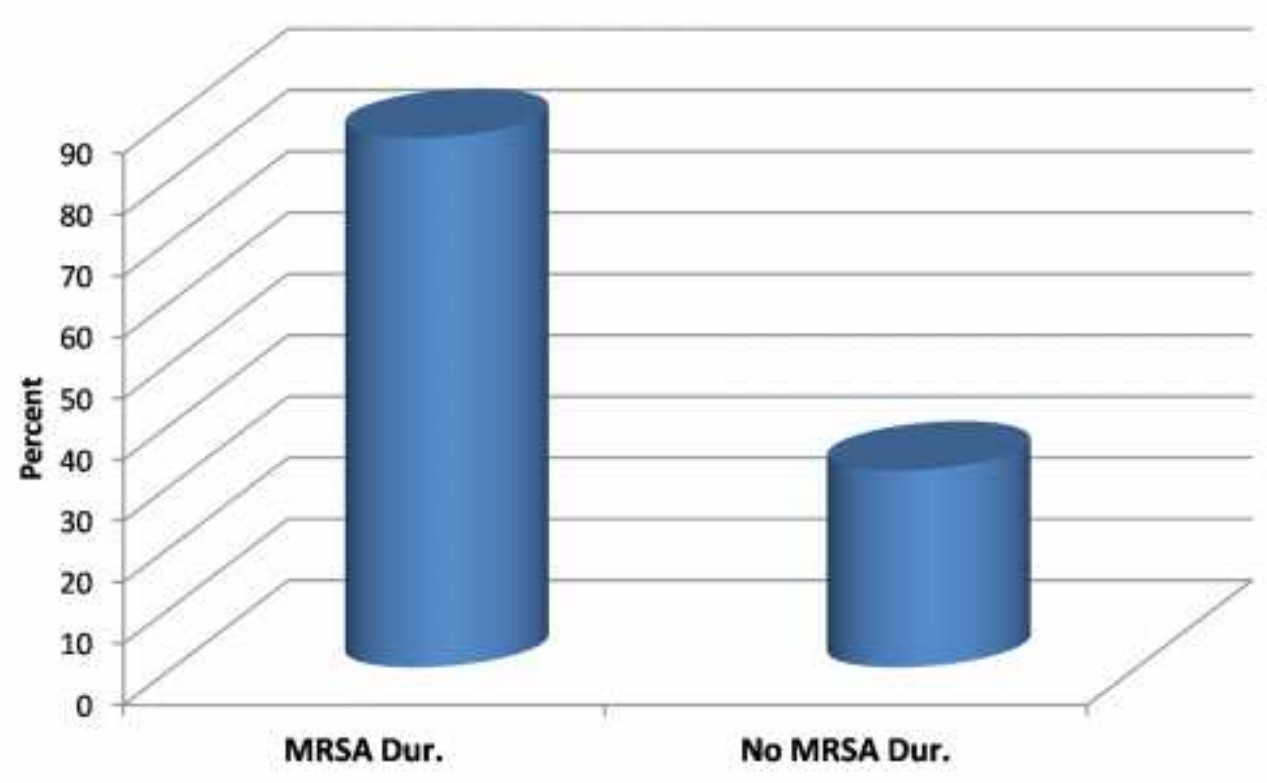

Figure (1): Percentage of presence of glucose in bronchial lavage between cases with MRSA during ICU stay and those without MRSA during ICU stay

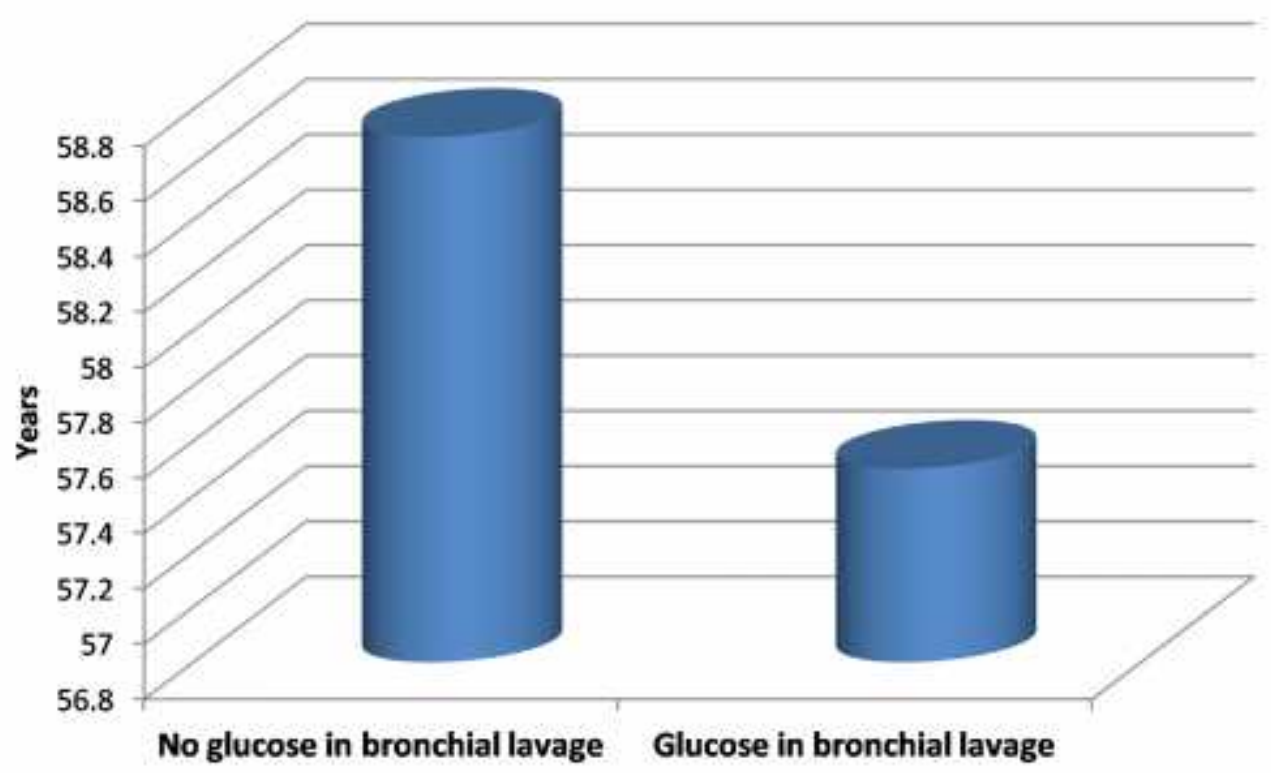

Figure (2): Mean age (years) between cases with glucose in bronchial lavage and those without glucose in bronchial lavage 


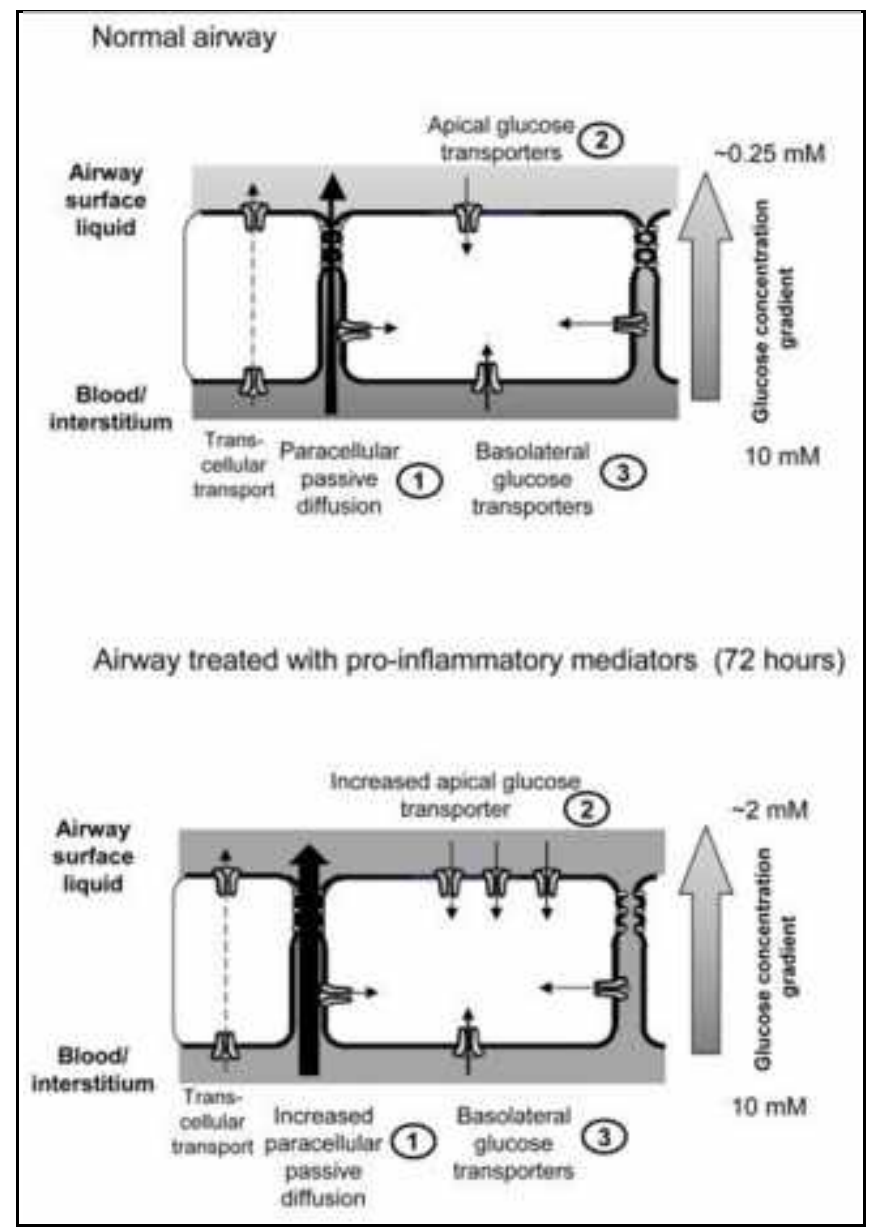

Fig. 3: Proposed mechanisms controlling glucose concentrations in airway surface liquid.

Normal airway: Under the normal conditions, glucose predominantly diffuses from the blood/interstitial fluid across the respiratory epithelium into airway surface liquid (ASL) via paracellular pathways and this is limited by the permeability of the epithelium. Apical and basolateral facilitative GLUT-mediated transport restricts glucose accumulation in ASL (there is little evidence for $\mathrm{Na}^{+}$-glucose co-transport in these airway cells). Glucose taken up into the cell is rapidly metabolised. This is critical to maintain low intracellular glucose concentrations which provide a driving force for glucose uptake via GLUT transporters. It also limits transcellular transport of glucose and predicts that ASL glucose concentrations equilibrate with intracellular glucose concentration.

Airway treated with pro-inflammatory mediators: Pro-inflammatory mediators reduce transepithelial resistance and increase paracellular diffusion of glucose across the epithelium into ASL, whilst apical GLUTmediated glucose transport and GLUT2 and 10 abundance were increased, insufficient to prevent a rise in glucose concentration in the ASL.

\section{Discussion}

MRSA pneumonia as with other infections caused by this organism is usually of nosocomial origin (Robotham et al, 2011) and it was suggested that St. aureus preferentially use glucose during the exponential phase of growth (Koo et al, 1996) glucose also affect the virulence of bacteria (Kutlu et al, 2014). The present study demonstrated the relation between the presence of glucose in the bronchial secretion and the development of MRSA infection. The factors associated with MRSA pneumonia are advanced age, prolonged hospitalization, gravity of the un- 
derlying diseases, especially lung diseases prior antibiotic therapy, and surgery or other invasive maneuvers; vesicular probe, central catheters, and endotracheal intubation (Bhayani et al, 2013). There were no differences in clinical findings, radiological in MRSA positive and MRSA negative in the present study which agreed with Wooten and Winston (2013) who analyzed patients with MRSA pneumonia and found these same risk factors for the acquisition of the infection, with the exception of the lung diseases.

Geerlings et al (2002) suggested that glucose may virulence the bacteria. As glucose is known to be important element in formation of biofilm in St. aureus protecting them from adverse environments, thus keeping bacteria pathogenicity by preventing clearance phase from host in many numbers of infections of them pneumonia. Where the presence of glucose in the airway may interfere with local immune process as virus replicate more rapidly in the lung of animal models and decrease the respiratory burst of alveolar macrophages impairing killing of bacteria this was interpreted by blocking the carbohydrate binding domains of proteins needed for recognition of attaching pathogens, which might explain the increase incidence of MRSA where glucose concentration in bronchial secretion was higher in the present study.. On the other hand, Kalsi et al. (2008) concluded that systemic hyperglycemia in animals models produce pro inflammatory cytokines including TNK and IL6 which in turn induce insulin resistance causing structural changes to animal lung .Although it was shown previously that hyperglycemia causes the appearance of glucose in the airway secretions and in those studies significant hyperglycemia were observed in patients with glucose in their bronchial secretion. However there were patients with glucose in bronchial secretion with normal or even low blood glucose levels at time of testing in our study hyperglycemia was not targeted (Cai et al, 2005). In humans the air way surface liquid glucose
(ASL) concentration was elevated when the airway epithelium is inflamed or when blood glucose is elevated. Nasal glucose concentration are undetectable (less than $1 \mathrm{mM}$ ) in healthy volunteers but detectable in $50 \%$ in patients with symptoms of viral rhinitis $(\sim 1 \mathrm{mM})$ and in $90 \%$ with diabetes mellitus $(\sim 4 \mathrm{mM})$ Breath glucose is elevated in people with lung inflammation due to cystic fibrosis $(2+-1.1 \mathrm{mM})$, with hyperglycemia due to diabetes mellitus $(1.2+-.07 \mathrm{mM})$ and to highest in people with both cystic fibrosis and diabetes mellitus; $4.0+-2.0 \mathrm{mM}$ (Baker et $a l, 2007)$. In intensive care patients with elevated airway surface liquid glucose concentration are more likely to have respiratory infection, particularly with methicillinresistant Staphylococcus aureus, than those with normal ASL glucose and this was the result in our study group. Number of possible explanation could explain the presence of glucose in airway secretion as it may enter the airway through leaking inflamed tissue (Fagon, 2011).

Philips et al. (2003) noted that volunteers with common cold glucose appear in their nasal secretion while they have normal blood level and disappear once symptom resolved. Another explanation is possible impairment of glucose clearance in critically ill patient (Popovich et al, 2013). Baker et al. (2007) found low level of glucose in airway secretion against the concentration gradient by active transport. Nathani et al. (2006) studied EBC glucose was higher in non-survivors compared to survivors. EBC glucose from infected patients was also higher than non-infected ones despite the presence of statistical significant difference between their serum glucose. They concluded that EBC glucose is potential marker of both infection/outcome in ARDS. They suggested that EBC collection might prove to be useful tool in guiding treatment and intervention in ARDS.

Despite these findings, the role of glucose in lung inflammation remains uncertain. Diabetes mellitus was found to be a negative 
predictor for acute respiratory distress syndrome (ARDS) secondary to sepsis. Although, when hyperglycemia was used as the predictor rather than a history of diabetes mellitus and the data were adjusted for confounding variables, there was no difference in the incidence of ARDS between groups (Moss et al, 2000). Wood et al, (2004) hypothesized that increased glucose concentrations in the airways increased the risk of chest infections, either by providing added nutrition for bacteria or by interfering with normal immune functions

As MRSA followed the detection of glucose in bronchial secretion in most cases, suggesting that glucose could have caused or promoted MRSA growth (Gordon and Lowly, 2008) which also was detected in the present study group, although it was unable to quantitative the MRSA load in individual patients and thereby differentiate between colonization and infection. It was suggested that St. aureus preferentially use glucose during the exponential phase of growth. Indeed, exit from this phase is in part determined by a lack of easily catabolisable carbohydrates (Somerville et al, 2002).

James et al. (2012) confirmed that the exposure of airway epithelium cell mono layers to pro inflammatory mediators increase ASL glucose concentration and was shown that the underlying mechanism is increased basolateral to apical paracellular glucose flux, which exceed compensatory up regulation of apical glucose transport. Because elevated ASL glucose concentration compromise the sterility of the lung and increase susceptibility to infection, insight into disruption of airway glucose homeostasis under inflammatory conditions could provide new therapeutic targets for lung infection (Pezzulo et al, 2011) The present study had documented a direct relation positive correlation between the presence of glucose in bronchial secretion of the critically ill patients and St. aureus infections, particularly MRSA. These results in agreed with Philips et al. (2005) who detected the glucose in bronchial secre- tion of 58 of 98 patients and those patients were more likely to have pathogenic bacteria than patients without glucose detected in bronchial secretions.

\section{Conclusion}

This simple, inexpensive and rapid technique in evaluating the bronchial secretion for the glucose might be opportunity to detect MRSA in incubated mechanically ventilated patients earlier so improve the clinicians diagnosis, patient hospital stay days and costs.

\section{References}

Baker, EH, Clark, N, Brennan, AL, Fisher, DA, Gyi, KM, et al, 2007: Hyperglycemia and cystic fibrosis alter respiratory fluid glucose concentrations estimated by breath condensate analysis. J. Appl. Physiol. 102:1969-75.

Bhayani, NH, Gupta, A, Dunst, CM, Kurian, AA, Reavis, KM, et al, 2013: Esophagectomies with thoracic incisions carry increased pulmonary morbidity. JAMA Surg. 148, 8:733-8.

Cai, D, Yuan, M, Frantz, DF, et al, 2005: Local and systemic insulin resistance resulting from hepatic activation of IKK-beta and NFkappaB. Nat. Med.11:183-9.

Fagon, JY, 2011: Biological markers and diagnosis of ventilator-associated pneumonia. Crit. Care 15:130-8.

Geerlings, SE, Meiland, R, van Lith, EC, et al, 2002: Adherence of type 1-fimbriated Escherichia coli to uroepithelial cells: more in diabetic women than in control subjects. Diabetes Care 25:1405-9

Gordon, RJ, Lowly, FD, 2008: Pathogenesis of methicillin- resistant Staphylococcus aureus infection. CID 46, 5:S350-9.

Hill, DM1, Schroeppel, TJ, Magnotti, LJ, Clement, LP, Sharpe, JP, et al, 2013: Methicillin resistant Staphylococcus aureus in early ventilator -associated pneumonia: cause for concern? 14, 60:520-4.

Hyllienmark, P, Martling, CR, Struwe, J, Petersson, J, 2012: Pathogens in the lower respiratory tract of intensive care unit patients: impact of duration of hospital care and mechanical ventilation. Scand. J. Infect. Dis. 44:444-52.

James, P, Garnett, TT, Nguyen, JD, Moffatt, ER, Pelham, KK, et al, 2012: Baines proinflammatory mediators disrupt glucose homeosta- 
sis in airway surface liquid. J. Immunol. 189:373-80.

Jones, RN, 2010: Microbial etiologies of hospital-acquired bacterial pneumonia and ventilatorassociated bacterial pneumonia. Clin. Infect. Dis. 51, 1:S81-7.

Kalsi, KK, Baker, EH, Medina, RA, Rice, S, Wood, DM, et al, 2008: Apical and basolateral localisation of GLUT2 transporters in human lung epithelial cells. Pflugers Arch. 456:9911003.

Kock, R, Becker, K, Cookson, B, et al, 2010: Methicillin-resistant Staphylococcus aureus (MRSA): Burden of disease and control challenges in Europe. Euro Surveill. 15, 41:19688.

Koo, SP, Yeaman, MR, Bayer, AS, 1996: Staphylocidal action of thrombin-induced platelet microbicidal protein is influenced by microenvironment and target cell growth phase. Infect. Immun. 64, 9:3758-64

Kutlu, M, Ergin, C, Bir, F, Hilmioğlu-Polat, S, Gümral, R, et al, 2014: Pulmonary $\mathrm{Mu}-$ cormycosis due to Lichtheimia ramosa in a patient with HIV Infection. Mycopathologia. 2014 Jun 13. [Epub ahead of print]

Moss, M, Guidot, DM, Steinberg, KP, Duhon, GF, Treece, P, Wolken R, et al, 2000: Diabetic patients have a decreased incidence of acute respiratory distress syndrome. Crit Care Med. 28, 7:2187-92.

Murray, PR, Washington, JA, 1975: Microscopic and bacteriologic analysis of expectorated sputum. Mayo Clin. Proc. 50:339-44

Nathani, N, Murphy, N, Manji. M, 2006: EBC glucose levels predict adverse outcome in ARDS ERS Programme 9:1116

Pezzulo, AA, Gutierrez, J, Duschner, KS, McConnell, KS, Taft, PJ, et al, 2011: Glucose depletion in the airway surface liquid is essential for sterility of the airways. PLoS One 6:e16166

Philips, BJ, Meguer, JX, Redman, J, et al, 2003: Factors determining the appearance of glucose in upper and lower respiratory tract secretions. Intensive Care Med. 29:220-10.

Philips, BJ, Redman, J, Brennan A, et al, 2005: Glucose in bronchial aspirates increases the risk of respiratory MRSA in intubated patients. Thorax 60:761-4.

Popovich, KJ, Hota, B, Aroutcheva, A, et al, 2013: Community-associated methicillinresistant Staphylococcus aureus colonization burden in HIV-infected patients. Clin. Infect. Dis. 56, 8:1067-74.

Robotham, JV, Graves, N, Cookson, BD, et al, 2011: Screening, isolation, and decolonisation strategies in the control of meticillin resistant Staphylococcus aureus in intensive care units: cost effectiveness evaluation. BMJ. 1 Oct 5;343:d5694. doi: 10.1136/bmj.d5694.

Shibabaw, A, Abebe, T, Mihret, A, 2014: Antimicrobial susceptibility pattern of nasal Staphylococcus aureus among Dessie Referral Hospital health care workers, Dessie, Northeast Ethiopia. Int. J. Infect. Dis. May 9. pii: S12019712(14)01480-5

Somerville, GA, Chaussee, MS, Morgan, CI, et al, 2002: Staphylococcus aureus aconitase inactivation unexpectedly inhibits postexponential-phase growth and enhances stationary-phase survival. Infect. Immun. 70:6373-82.

Tacconelli, E, De Angelis, G, 2009: Pneumonia due to methicillin-resistant Staphyloccus aureus: Clinical features, diagnosis and management. Curr. Opin. Pulm. Med. 15, 3:218-22.

Van den Berghe, G, Wouters, P, Weekers, F, et al, 2001: Intensive insulin therapy in the critically ill patients. N. Engl. J. Med. 345:1359-67.

Wood, DM, Brennan, AL, Philips, BJ, et al, 2004: Effect of hyperglycaemia on glucose concentration of human of human secretion Clin. Sci. (Lond); 106:527-33

Wooten, DA, Winston, LG, 2013: Risk factors for methicillin-resistant Staphylococcus aureus in patients with community-onset and hospitalonset pneumonia. Respir. Med. 107, 8:1266-70. 